\title{
Teneurs en NOx du lait et des produits laitiers : aspects technologiques et biochimiques
}

\author{
par \\ M. F. GUINGAMP et G. LINDEN
}

\section{Sommaire}

I. Introduction $\ldots \begin{array}{lllllllllllllllllllll} & \ldots & \ldots & \ldots & \ldots & \ldots & \ldots & \ldots & \ldots & \ldots & \ldots & \ldots & \ldots & \ldots & \ldots & \ldots & \ldots & \ldots & 427\end{array}$

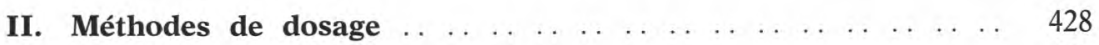

II.1. Dosage des nitrites .. . . . . . . . . . . . . . . . . . . . . . . . 428

II.1.1. Méthodes spectrométriques . . . . . . . . . . . . . . . . . . . 428

II.1.2. Autres méthodes .. . . . . . . . . . . . . . . . . . . . . . . . . . . . 429

II.2. Dosage des nitrates . . . . . . . . .

II.2.1. Méthodes spectrométriques . . . . . . . . . . . . . . . . . . . 429

II.2.2. Méthodes électrochimiques . . . . . . . . . . . . . . . 430

II.2.3. Méthodes chromatographiques . . . . . . . . . . . . . . . 430

III. Harmonisation des méthodes et législation $\ldots \ldots \ldots \ldots$. . . . . 431

III.1. Harmonisation des méthodes .. . . . . . . . . . . . . . . 431

III.2. Législation . .

IV. Les teneurs en nitrates-nitrites du lait et des produits laitiers 432

V. Influence des traitements thermiques sur les variations des

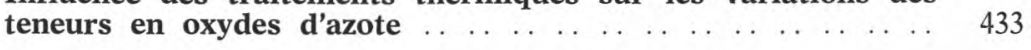

VI. Aspects biochimiques et toxicologiques . . . . . . . . . . . . . . . . . 4434

VI.1. Réactivité avec les constituants biochimiques .. . . . 435

VI.1.1. Nitrites et lipides . . . . . . . . . . . . . . . . . . . . . . . . . . . . . 435

VI.1.2. Nitrites et glucides .. . . . . . . . . . . . . . . . . . . . . . 436

VI.1.3. Nitrites et protéines . . . . . . . . . . . . . . . . . . . . . . 436

VI.2. Toxicité des nitrates et des nitrites et formation de 436

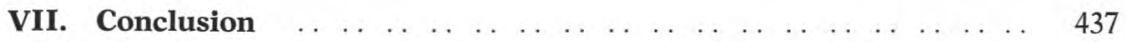

Laboratoire de Biochimie Appliquée, Université de Nancy I, B.P. 239 - 54506 Vandœuvre-lès-Nancy cedex. 


\section{Résu mé}

Le dosage des nitrates et/ou des nitrites s'effectue essentiellement par des méthodes spectrométriques ou électrochimiques. Ces dernières sont encore mal adaptées aux produits complexes. Les techniques spectrométriques, bien que couramment employées et comparées entre elles, ne donnent pas entière satisfaction, car dans les milieux complexes, il est difficile de savoir quels NOx, libres ou liés ont été dosés.

La Commission Générale d'Unification des méthodes d'analyse a adopté la méthode spectrométrique à $540 \mathrm{~nm}$ en présence de sulfanylamide et de naphtylèthylènediamine (N.E.D.) après réduction des nitrates sur une colonne de cadmium. Les teneurs limites de divers produits laitiers sont rappelées.

Les laits crus contiennent très peu de nitrates-nitrites, mais après les traitements thermiques industriels, les teneurs des produits laitiers sont plus élevées. Des expériences ont montré l'importance des équilibres $\mathrm{NO}_{\mathrm{x}}$ libres $\leftrightharpoons \mathrm{NO}$ x liés ; équilibres qui sont perturbés par les traitements technologiques mais aussi par certaines étapes analytiques. Ainsi tout résultat d'analyse devrait être accompagné de l'historique de l'échantillon et des conditions de réalisation du dosage.

Certains oxydes d'azote, le nitrite en particulier, présentent une forte réactivité vis-à-vis des constituants biochimiques : glucides, lipides, et surtout protéines. L'action du nitrite sur les amines secondaires ou tertiaires est connue, elle peut se dérouler dans la chaîne alimentaire mais aussi dans l'organisme humain. Il a été trouvé des composés nitrosés en faible quantité dans certains produits laitiers.

\section{Mots clés :}

Nitrates - Nitrites - Produits laitiers - Méthodes de dosage - Teneurs -

Traitements technologiques Réactivité biochimique.

\section{S u m m a ry}

NOx LEVELS IN MILK AND MILK PRODUCTS: BIOCHEMICAL AND TECHNOLOGICAL FEATURES

Spectrometric or electrochemical methods are generally used for nitrate or nitrite determination. Electrochemical methods are not really convenient for the complex products. Spectrometric techniques, although commonly used do not give satisfaction because of the difficulty in determining whether free or bound $N O_{x}$ of the foodstuff is so detected. 
The Committee of methods analysis harmonisation has chosen the spectrometric method calling for a nitrate reduction on a cadmium column in the presence of sulfanylamid and N.E.D. (at $540 \mathrm{~nm}$ ). The limiting values for some dairy products are recalled.

Nitrates levels in raw milks are very low, they are greater after industrial thermal processings. Some experiments have pointed out the importance of the free $N O_{x} \leftrightharpoons$ bound $N O_{x}$ equilibriums. However these are disturbed by the technological processings and also by certain analytical steps. Valid results can be given only when the foodstuff's history and the analytical techniques are well-known.

Some nitrogenous oxides, especially the nitrites, are very reactive with biochemical compounds such as glucids, lipids and proteins. The nitrite reaction with secondary or teritary amines is well-known; it can take place in foods and in the human body. Nitroso compounds have been found with low levels in certain dairy products.

Key words:

Nitrates - Nitrites - Dairy products - Determination methods - Occurrence -

Technological processings - Biochemical reactivity.

\section{INTRODUCTION}

L'emploi des nitrates et des nitrites est interdit en France dans l'industrie fromagère. Il a été montré que les teneurs en nitratesnitrites des laits crus sont en général très faibles, quelle que soit l'alimentation des vaches. Et pourtant, quelques comptes rendus d'analyse font état de teneurs anormalement élevées : $2 \mathrm{mg} / \mathrm{l}$ dans des laits industriels, 50 à $100 \mathrm{mg} / \mathrm{kg}$ dans les laits en poudre, plus de $100 \mathrm{mg} / \mathrm{kg}$ dans des poudres de lactosérums.

Il existe de nombreuses méthodes de dosage des nitrates et/ou des nitrites : des méthodes spectrométriques surtout, des méthodes électrochimiques (plus rapides mais encore mal adaptées à la complexité des produits laitiers) et des méthodes enzymatiques.

Quelle que soit la méthode employée, il convient de souligner que les oxydes d'azote $\left(\mathrm{NO}_{\mathrm{x}}\right)$ peuvent être impliqués dans des équilibres réactionnels, tant lors de la préparation de l'échantillon que lors des transformations technologiques du lait et des produits laitiers.

Après une revue critique des principales méthodes de dosage, nous évoquerons la question de l'harmonisation des protocoles d'analyse et de l'aspect législatif. Dans un deuxième temps, nous commenterons les taux de nitrates-nitrites relevés dans les laits et différents produits laitiers et essayerons d'expliquer les causes de variabilité de ces teneurs. 


\section{METHODES DE DOSAGE}

La plupart des protocoles analytiques comportent une extraction aqueuse en milieu alcalin pour éviter la destruction des nitrites et une purification destinée à éliminer la matière grasse et les protéines. Des techniques variées sont utilisées pour purifier et clarifier les extraits qui vont servir au dosage des nitrates et des nitrites (Brugel, 1980 ; Frouin et coll., 1980).

La technique la plus utilisée est la défécation. Il existe plusieurs réactifs défécants ; mais selon Usher et Telling (1975), l'acétate de zinc + ferrocyanure de potassium permet d'obtenir en milieu alcalin des filtrats très limpides. Mais l'échange d'ions, la chromatographie sur couche mince et l'électrodialyse ont également été employés.

Le chauffage, au cours de ces étapes, doit être réduit au minimum, afin de ne pas perturber les équilibres nitrites-nitrates/milieu réactionnel. Rougié, Noël et Goutefongea (1980) ont montré que des températures de chauffage et des temps d'extraction différents modifiaient le taux d'extraction du nitrite ajouté lors de la fabrication de jambons ( $1 \mathrm{~h}$ à $20^{\circ} \mathrm{C}: 22 \% ; 1 / 2 \mathrm{~h}$ à $\left.100^{\circ} \mathrm{C}: 57 \%\right)$. Le dosage des nitrites et/ou des nitrates s'effectue ensuite sur le surnageant obtenu.

\section{II.1. Dosage des nitrites}

\section{II.1.1. Méthodes spectrométriques}

La principale réaction utilisée est la formation d'un colorant azoïque, selon le schéma (Frouin et coll., 1980).

Première réaction :

$\mathrm{NO}_{2}^{-}+$amine primaire aromatique $\stackrel{\mathrm{CH}_{3} \mathrm{COOH}}{\longrightarrow}$ sel de diazonium.

Deuxième réaction :

Sel de diazonium + phénol $\stackrel{\mathrm{pH} \text { basique }}{>}$ Colorant azoïque $=$ réaction de Zambelli

ou

Sel de diazonium + amine aromatique $\stackrel{\mathrm{pH} \text { acide }}{\longrightarrow}>$ Colorant azoïque $=$ réaction de Griess.

Les variantes de ces méthodes spectrométriques reposent essentiellement sur le type de composé aromatique utilisé dans la première ou la deuxième réaction (Brugel, 1980).

D'autres méthodes spectrométriques sont possibles mais sont peu utilisées :

Réactions avec :

- l'acide chromotropique (Barbieri et coll., 1979) ; 
- l'antipyrine utilisée par Frouin et coll. (1976b) ;

- la 5-aminofluoresceine et formation de composés fluorescents (citée par Frouin et coll., 1980).

Réaction de :

- Treadwell avec le sulfite de sodium utilisée par Frouin et coll. (1976a).

\section{II.1.2. Autres méthodes}

Certaines techniques prévoient le dosage des nitrites (et surtout des nitrates) par polarographie (Huynh, Jacquet et Silberzahn, 1969 ; Cox et Brajter, 1979; Branca, Ricottilli et Spagnolini, 1980) ou par électrodes spécifiques, mais ces méthodes sont difficilement applicables aux produits complexes (produits laitiers ou viandes) car les causes d'interaction sont nombreuses.

La chromatographie en phase gazeuse avec détecteur à capture d'électrons permet, elle aussi, le dosage des nitrites après réaction de ceux-ci avec des dérivés benzéniques (Funazo, Tanaka et Shono, 1980) ou avec du l-hydrazinophtalazine (Tanaka et coll., 1981). Notons également l'utilisation possible de détecteur à ionisation de flamme (Toyoda et coll., 1978).

Enfin, nous allons voir dans le paragraphe suivant que les nitrites peuvent également être dosés par chromatographie liquide haute pression et par chimiluminescence.

\section{II.2. Dosage des nitrates}

On fait appel essentiellement à des méthodes spectrométriques ou électrochimiques.

\section{II.2.1. Méthodes spectrométriques}

Elles sont basées (Usher et Telling, 1975) sur :

- la formation d'un composé phénolique nitré ; on utilise souvent le xylénol comme composé phénolique. Cette méthode, recommandée en Italie, suscite de nombreuses réserves (Amariglio, 1982 ; Guingamp, Schwartz et Linden, 1978). Elle ne peut être automatisée et Cserhati (1973), Laskowski et Bierska (1974a) pensent que la mauvaise reproductibilité des résultats s'explique essentiellement par la présence d'une distillation dans le protocole opératoire ;

- l'oxydation par les nitrates d'un composé phénolique, tel que la brucine ou la diphénylamine; méthodes utilisées ou testées par Laskowski et Bierska (1974b) ou par Mrowetz et Klostermeyer (1977a) ;

- la réduction des nitrates en nitrites puis dosage de ces derniers.

Cette réduction peut être réalisée de différentes façons :

- Soit par voie enzymatique, à l'aide de nitrates réductases de différentes origines (Sen, Carr et Klatt, 1976; Kiang, Kuan et 
Guilbaut, 1978a et b). Ces méthodes ont été peu testées dans les milieux complexes.

- Soit par voie chimique. Ce sont les techniques les plus utilisées, en particulier celles faisant appel au cadmium, ou à "l'alliage cadmiumcuivre ". De nombreux auteurs les ont utilisées ou comparées à d'autres dans le domaine des produits laitiers (Manning, Coulter et Jenness, 1968 ; Mac Kay, 1974 ; Hamilton, 1976 ; Kreisner et Fink, 1978 ; Mrowetz et Klostermeyer, 1977a et b ; Guingamp, Schwartz et Linden, 1978 ; Amariglio et Imbert, 1980).

Cette réduction est influencée par la granulométrie et le "vieillissement » du métal, et par le pH du milieu (Klein, 1982). Elle est sans doute accompagnée de réactions secondaires très mal connues qui perturbent les résultats obtenus si le milieu analysé est complexe (Frouin et coll., 1976a et b ; Frouin et coll., 1980). Ces phénomènes seront revus plus loin.

Le réducteur peut être aussi le sulfate d'hydrazine (Nijhuis, Heeschen et Blüthgen, 1979).

Ces techniques chimiques de réduction, suivies d'une réaction colorée (réactif de Griess) se prêtent fort bien à l'analyse en flux continu.

\section{II.2.2. Méthodes électrochimiques}

- électrode spécifique : bien adaptée aux dosages des milieux simples et des végétaux. Cette technique a donné lieu à de nombreuses publications (Choi et Fung, 1980 ; Kaneda, Kanamori et Iwaïda, 1977 ; Lange, 1981 ; Liedtke et Meloan, 1976 ; Pfeiffer et Smith, 1975 ; Sweetsur et Wilson, 1975 ; Zimmermann et Von Lengerken, 1980a et b) ;

- polarographie (Cox et Litwinsky, 1979 ; Huynh, Jacquet et Silberzahn, 1969 ; Branca, Ricotilli et Spagnolini (1980) mise au point dans les milieux simples et les végétaux ;

- chimiluminescence : bien que cette technique nécessite un détecteur T.E.A. (Thermal Energy Analyser) d'un prix élevé, elle présente l'avantage de pouvoir doser, dans une matrice, les nitrites, les composés N-nitrosés et les nitrates par additions successives d'acide acétique, d'acide bromhydrique et de chlorure de titane (Walters et coll., 1980 ; Cox, 1980). Le schéma des réactions est le suivant :

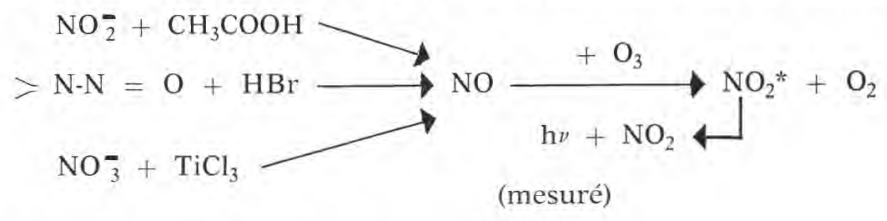

\section{II.2.3. Méthodes chromatographiques}

Par chromatographie en phase gazeuse avec détecteur à capture d'électrons, les nitrates peuvent être dosés après formation du nitro- 
xylénol (Wu et Saschenbrecker, 1977; Toyoda et coll., 1978) ou du nitro-2-Sec. butylphénol (Tanaka, Nose et Iwasaki, 1982).

Iskandarani et Pletrzyk (1982) utilisent une chromatographie liquide haute pression pour la séparation et la détermination des nitrates et nitrites basée sur un modèle d'interaction d'ions double couche. Un absorbant copolymérique non polaire (polystyrène divinyl benzène) est utilisé comme phase stationnaire et la séparation est effectuée par un contrôle de l'équilibre intervenant entre le composé, la phase stationnaire, un sel de tétra-alkylammonium et son coanion. En employant une colonne échangeuse d'anions, Söderhjelm (1982) a dosé par H.P.L.C. les nitrates dans la poudre de lait, de lactosérum ou dans du fromage avec une limite de détection de $1 \mathrm{mg}$ par $\mathrm{kg}$.

Pour nous résumer, on peut affirmer qu'avec de nombreuses méthodes il est aisé d'obtenir un résultat précis et significatif dans un milieu simple ou peu chargé. Dans les milieux complexes tels que les produits laitiers, on ne connaît pas toujours très bien, comme on le verra par la suite, le composé ayant réagi. Il est difficile de savoir si l'on a dosé les nitrites et nitrates libres ou divers oxydes d'azote liés mais libérab'es dans des conditions analytiques bien déterminées (chauffage, défécation, passage sur colonne de cadmium...).

\section{HARMONISATION DES METHODES ET LEGISLATION}

\section{III.1. Harmonisation des méthodes}

Depuis 1969, l'Organisation Internationale de Normalisation (I.S.O.) s'est penchée sur le problème du dosage des nitrates-nitrites dans les produits laitiers. Un groupe de travail restreint I.S.O./F.I.L./ A.O.A.C. est alors créé et il est chargé de faire l'inventaire des techniques et de tester quatre d'entre elles (Amariglio, 1982), à savoir :

- l'oxydation de la diphénylamine ;

- la nitration du 2.4 xylénol ;

- la formation d'un colorant azoïque par réaction du nitrite avec la sulfanylamide puis la $\mathrm{N}_{1}$-Naphtyléthylènediamine (N.E.D.) ; le nitrite étant obtenu par réduction du nitrate après passage sur une colonne de cadmium ; cifique.

- la détermination potentiométrique à l'aide d'une électrode spé-

Reste le problème des échantillons de référence. De nombreux produits laitiers peuvent être utilisés, mais pour des raisons de stabilité et d'homogénéité, le lait en poudre est un matériel de premier choix. Le bureau de références (B.C.R.) créé en 1972 auprès de la C.E.E. a un programme pluri-annuel de mise au point d'échantillons de réfé- 
rence, dont celui d'une poudre de lait contenant une trentaine de cations et d'anions parmi lesquels on relève les nitrates.

Après des recherches bibliographiques et des analyses circulaires qui ont duré plusieurs années, la Commission Générale d'Unification des méthodes d'analyse a adopté la méthode de dosage des nitrites et des nitrates par dosage spectrométrique à $540 \mathrm{~nm}$ en présence de sulfanylamide et de N.E.D., après réduction des nitrates par passage sur colonne de cadmium.

\section{III.2. Législation}

Un certain nombre d'arrêtés (1976-07.19; 1978-03.30;1980-01.16 ; 1981-01.05) précisent les teneurs limites en nitrates-nitrites de divers produits laitiers et aliments lactés. Nous les avons consignées dans le tableau ci-dessous :

\begin{tabular}{l|c}
\hline \multicolumn{1}{c}{ Type de produit } & Teneurs maximales en $\mathrm{NO}_{-3}$ (et $\mathrm{NO}_{-2}$ ) \\
\cline { 2 - 2 } $\begin{array}{l}\text { Aliments de régime destinés aux nour- } \\
\text { rissons de moins de } 3 \text { mois }\end{array}$ & $50 \mathrm{mg} / \mathrm{kg}$ \\
Aliments lactés diététiques & $\left\{\begin{array}{c}50,4 \mathrm{mg} / \mathrm{kg} \text { produit complet } \\
37 \mathrm{mg} / \mathrm{kg} \text { produit écrémé } \\
\text { ou } 0,24 \mathrm{mg} / 100 \mathrm{~K} \text { joules }\end{array}\right.$ \\
Laits et fromages & non précisées \\
Lactosérum hydrolysé & $50 \mathrm{mg} / \mathrm{kg} / \mathrm{E} . \mathrm{S}$. \\
Aliments des animaux & $\mathrm{NO}-\overline{2}: 0,1 \mathrm{mg} / \mathrm{kg}$ \\
(aliments complets) & $\mathrm{NO}-\overline{3}: \mathrm{non}$ précisées \\
\hline
\end{tabular}

Les méthodes internationales (F.I.L./I.S.O.) de dosage des nitritesnitrates ont été publiées en 1980. Les protocoles s'appliquent aux fromages, laits secs, sérums de fromagerie et lactosérums en poudre et les normes provisoires sont référenciées en fin d'article.

\section{TENEURS EN NITRATES-NITRITES DU LAIT ET DES PRODUITS LAITIERS}

Les teneurs en nitrates-nitrites des laits crus sont faibles (Nijhuis et coll., 1982 ; Mahieu, Luquet et Mouillet, 1976-1977) et dépendent peu de l'alimentation des vaches (Rémond, 1975). Elles sont de l'ordre de 0,3 à $0,5 \mathrm{mg} / 1$ et ne dépassent $1 \mathrm{mg} / 1$ que très exceptionnellement (Mahieu et coll., 1980 ; Mahieu et coll., 1982). 
Le Laboratoire central d'Hygiène Alimentaire a procédé à de nombreuses analyses (Amariglio et Imbert, 1980), qui, si elles ne sont pas tout à fait représentatives de l'ensemble de la production française, montrent cependant que :

- en 1979, $82 \%$ des laits secs ont une contamination inférieure à $20 \mathrm{mg} / \mathrm{kg}$ (contre $94 \%$ en 1978) ;

- $56 \%$ des lactosérums ont des teneurs inférieures à $50 \mathrm{mg} / \mathrm{kg}$ (70\% en 1978) ;

- $58 \%$ des aliments d'allaitement renferment moins de $30 \mathrm{mg} / \mathrm{kg}$ $(87 \%$ en 1978) ; en 1979).

- les fromages sont peu contaminés (92\% inférieurs à $10 \mathrm{mg} / \mathrm{kg}$

Nous allons revenir sur ces valeurs et leur variabilité dans le paragraphe suivant.

\section{INFLUENCE DES TRAITEMENTS THERMIQUES SUR LES VARIATIONS DES TENEURS EN OXYDES D'AZOTE}

A ces données analytiques brutes, il faut ajouter qu'on a relevé de nombreuses discordances dans les résultats. Ces variations sont, comme nous allons le voir, liées la plupart du temps aux traitements industriels ou analytiques qu'ont subis les produits (Resmini et Volonterio, 1973 ; Przybylowski et Kisza, 1982).

Ainsi, les teneurs des poudres de lactosérum sont plus fortes que celles des sérums liquides correspondants (Görner, Hluchan et Antalike, 1978). Des essais pilotes ont permis de montrer qu'une partie de cet enrichissement apparent en nitrates se produit lors de l'étape de concentration du sérum et qu'il est sous la dépendance de la température (Guingamp, Schwartz et Linden, 1978).

De même, nous avons constaté que la pasteurisation et la stérilisation multipliaient la teneur des composés dosés comme nitrates par un facteur pouvant être égal à 4 (Guingamp et Linden, 1980).

Par ailleurs, Rothery (1968) et Wheeler (1980) ont montré que les oxydes d'azote (NOx) formés lors de l'atomisation à chauffage direct, peuvent réagir avec les groupements aminés des protéines. Ces oxydes d'azote sont dosés comme des nitrates ou des nitrites. Leur concentration dans les produits secs varie avec le type de chauffage choisi (Harding et Gregson, 1978). Manning, Coulter et Jenness (1968) ont observé que l'atomisation avec chauffage indirect de l'air n'augmentait que faiblement les teneurs en nitrates des laits écrémés ou entiers. Cependant, il a été montré que pour une même concentration de $\mathrm{NO}_{\mathrm{x}}$ dans les gaz réchauffés, le taux de produits dosés comme 
nitrates varie avec l'indice " $\mathrm{x}$ " de $\mathrm{NO}_{\mathrm{x}}$ et surtout avec le $\mathrm{pH}$ du produit séché ; un produit légèrement acide (lait ou lactosérum) fixe peu de $\mathrm{NO}_{x}$ (1 à $2 \%$ ) alors qu'un produit basique (caséinate de sodium) peut en fixer des quantités très importantes (50 à $60 \%$ ).

En outre, des expériences de chauffage, de congélation-décongélation du lait ou de lactosérums de fromagerie ont montré l'importance des équilibres $\mathrm{NO}_{2}-\mathrm{NO}_{3}$ libres $\leftrightharpoons \mathrm{NO}_{2}-\mathrm{NO}_{3}$ liés au substrat (Guingamp et Linden, 1981).

Chopin (1983) a étudié la cinétique de fixation des nitrates sur la caséine bovine en solution. La teneur en nitrates libres (ajoutés ou non) des solutions de caséine bovine est minimale à $\mathrm{pH}$ neutre et pour une température d'environ $30^{\circ} \mathrm{C}$. En outre, la fixation des nitrates par unité de masse protéique est d'autant plus faible que la concentration en caséine est élevée.

Ces équilibres sont non seulement perturbés par les traitements technologiques mais également par certaines étapes analytiques. Par exemple, le passage en milieu réducteur libère des oxydes d'azote dosés comme nitrates au cours de la réaction colorée en présence du réactif de Griess modifié.

Des taux anormalement élevés en nitrates-nitrites peuvent aussi être bien sûr d'origine accidentelle ; par exemple, un mauvais rinçage du matériel après lavage à l'acide nitrique, une contamination de lactosérums par l'eau de lavage de certains caillés (Guingamp, Linden et Alais, 1982).

En résumé, la validité des dosages de nitrites-nitrates dans les produits laitiers ne peut être prise en compte que si l'on connaît l' « historique » de l'échantillon et l'intégralité des conditions de réalisation du dosage.

\section{ASPECTS BIOCHIMIQUES ET TOXICOLOGIQUES}

Ces phénomènes ont été constatés et étudiés dans d'autres systèmes alimentaires comme les produits carnés par exemple (Rougié, Noël et Goutefongea, 1980 ; Frouin et coll., 1980). Cassens et coll. (1976) ont montré que certains oxydes d'azote présentent une forte réactivité vis-à-vis des grandes classes de constituants biochimiques mais ce sont les protéines qui sont de loin les plus touchées.

Parmi les effets nocifs du nitrite, on peut citer :

- la méthémoglobinémie ;

- la diminution de la phosphorylation oxydative et l'inhibition d'enzymes microsomales (Lowy et Manchon, 1976) ; 
- la diminution de l'efficacité vitaminique de la ration alimentaire (Lhuissier, Suschetet et Causeret, 1976) ;

- la réactivité vis-à-vis de différents constituants biochimiques.

Nous allons développer ce dernier type de réactivité car c'est sans doute celui qui présente le plus d'intérêt dans le lait et les produits laitiers.

\section{VI.1. Réactivité avec les constituants biochimiques}

Toutes les grandes familles de constituants biochimiques sont concernées et cette réactivité multidirectionnelle se trouve résumée dans la figure 1.

\section{VI.1.1. Nitrites et lipides}

Woolford et Cassens (1977), Goutefongea, Cassens et Woolford (1977) montrent dans les produits de charcuterie qu'un faible pourcentage de nitrite (de l'ordre de $5 \%$ maximum) se trouve fixé dans la fraction lipidique du tissu adipeux. Ces auteurs, par incubation d'acides gras et de mono et triglycérides présentant différents degrés d'insaturation, ont montré que la fixation du nitrite est en relation avec le nombre de doubles liaisons. Kurechi et Kikugawa (1979)

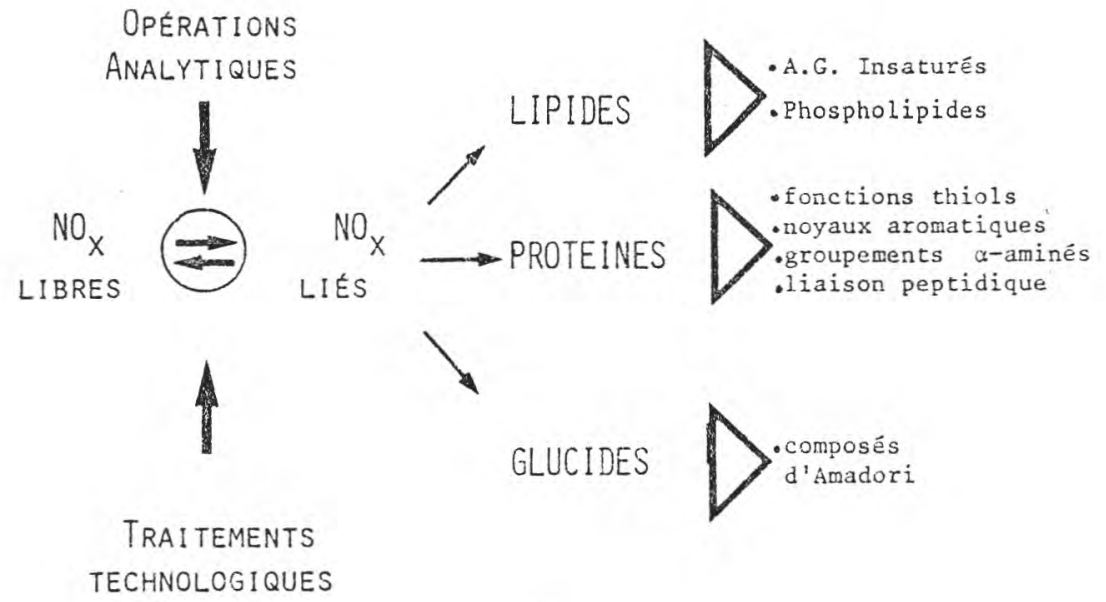

fig. 1

Réactivité des oxydes d'azote avec les constituants biochimiques.

Nitrogenous oxyde reactivity with biochemical compounds. 
montrent que les réactions des nitrites avec les lipides diminuent la formation de nitrosamines, ce qui voudrait dire que la réaction lipides- $\mathrm{NO}_{2}^{-}$se ferait plus facilement que la réaction amines- $\mathrm{NO}_{2}^{-}$.

\section{VI.1.2. Nitrites et glucides}

On ne constate pas de variations de la teneur en nitrates d'une solution à laquelle on ajoute du lactose. Cependant, un mélange de caséine et de lactose en solution ayant subi une réaction de Maillard, fixe plusieurs formes d'oxyde d'azote (Chopin, 1982). Ces résultats peuvent être expliqués par le fait que les composés d'Amadori fixent les formes NOx (Zaugg, 1961 ; Moll, 1983).

\section{VI.1.3. Nitrites et protéines}

A côté de son action sur la partie hémique des protéines, le nitrite peut réagir avec des groupements sulfydryles mais les produits obtenus sont instables (Kubberod, Cassens et Greaser, 1974). La réaction avec les acides aminés aromatiques est chimiquement établie (Nakai et coll., 1978 ; Woolford et coll., 1976) mais son importance effective dans les milieux complexes est à préciser (Ito, Cassens et Greaser, 1979).

Enfin, Bonnett et Holleyhead (1974) pensent que la liaison peptidique peut intervenir comme site de fixation, mais ils n'ont pu le démontrer clairement. En utilisant un modèle simple, la polyglycine, des travaux récents ont permis de montrer l'intervention des liaisons peptidiques dans la fixation des nitrites par les protéines (Guingamp et Linden, 1983 ; Chopin, 1983). De même, Pollock (1981) a étudié des dérivés nitrosés obtenus après action du nitrite sur divers dipeptides.

\section{Vi.2. Toxicité des nitrates et des nitrites et formation de nitrosamines}

Depuis plusieurs années, les teneurs croissantes en nitrates-nitrites des aliments et des boissons (Deschamps, 1980 ; Voilliot, Mère et Degrimal, 1980 ; Melet et coll., 1980), posent un problème important en raison de leurs effets toxiques. En 1976, la F.A.O. et l'O.M.S. ont établi une dose journalière admissible (D.J.A.) de $3,65 \mathrm{mg}$ d'ion $\mathrm{NO}_{3}^{-}$ et de $0,133 \mathrm{mg}$ d'ion $\mathrm{NO}_{2}^{-}$par $\mathrm{kg}$ de poids corporel pour l'homme, et une DL.50 (chez le rat) de $2,3 \mathrm{~g} / \mathrm{kg}$ pour $\mathrm{NO}_{3}^{-}$et $0,05 \mathrm{~g} / \mathrm{kg}$ pour $\mathrm{NO}_{2}^{-}$.

Il est admis (De Saint-Blanquat, 1980) que dans le couple $\mathrm{NO}_{3} / \mathrm{NO}_{2}$ c'est surtout le nitrite qui pose un problème toxicologique, probablement à cause de sa réactivité chimique particulière. Il peut réagir avec de nombreux groupements fonctionnels d'origine alimentaire par l'intermédiaire de ses propriétés oxydantes ou réductrices.

Si l'on a imposé des teneurs limites en nitrates, c'est qu'ils peuvent très facilement être transformés en nitrites, soit dans l'aliment lui-même en présence de micro-organismes, soit chez l'homme, grâce 
à sa flore digestive bactérienne (Fritsch, Klein et De Saint-Blanquat, 1980 ; Klein et coll. 1978).

La réactivité du nitrite sur les amines secondaires ou tertiaires est connue ; elle s'écrit :

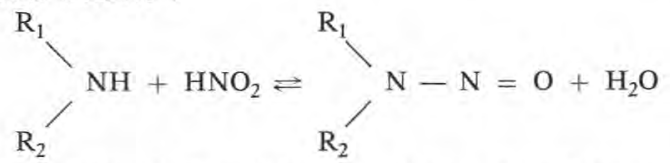

Cette réaction peut se dérouler dans l'environnement au sens large du terme, plus particulièrement dans la chaîne alimentaire et dans l'organisme humain (Klein et coll., 1976). Knowles et coll. (1974) ont également mis en évidence à $\mathrm{pH}$ gastrique la formation de composés C-nitrosés à partir de nitrosoaminoacides.

De nombreux aliments ont fait l'objet d'analyses et il a été trouvé des nitrosamines en quantités plus ou moins importantes dans les produits de charcuterie, dans les bières et même dans les fromages (Klein et Debry, 1980). Keshavarz-Dehno (1979) et Klein et coll. (1980) ont montré l'apparition de nitrosamines au cours de l'affinage de fromages expérimentaux de type Camembert, fabriqués à partir de lait nitraté et ensemencés à l'aide d'une souche de Penicillium Camemberti.

Les micro-organismes catalyseraient l'apparition de nitrosamines car leur formation est peu probable dans les milieux peu acides que sont en général les aliments.

Sen et coll. (1978) relèvent la présence dans un certain nombre de fromages importés du Canada, de nitrosodiméthylamine (N.D.M.A.), de nitrosodiéthylamine (N.D.E.A.) et de nitrosopipéridine (N.P.I.P.) aux taux de 2 à $20 \mu \mathrm{g} / \mathrm{kg}$.

Chez différents animaux de laboratoire, l'action cancérigène de ces composés nitrosés n'est plus à démontrer. Ils induisent des tumeurs au niveau d'un certain nombre d'organes, le site d'action dépendant essentiellement de la structure du composé.

\section{CONCLUSION}

Les laits crus contiennent en général peu de nitrates-nitrites. Après les traitements thermiques industriels, les produits laitiers peuvent en renfermer des quantités plus importantes.

Les contaminations accidentelles étant écartées, ces augmentations pourraient s'expliquer par le déplacement des équilibres $\mathrm{NO}_{\mathrm{x}}$ libres $\leftrightharpoons \mathrm{NO}_{\mathrm{x}}$ liés, lors de traitements technologiques ou d'opérations analytiques.

De surcroît, les oxydes d'azote peuvent réagir avec de nombreux constituants biochimiques présents dans le lait et les produits laitiers. Même si dans ce type de produits le problème des nitrites-nitrates 
n'est pas aussi aigu que dans d'autres secteurs alimentaires, il mérite cependant d'être suivi avec vigilance et rigueur.

\section{Remerciements}

Nous remercions $\mathrm{M}$. le $\operatorname{Pr} \mathrm{C}$. Alais pour ses précieux conseils dans la rédaction du manuscrit et MM. D. Klein et D. Lorient pour leur aide amicale.

\section{Bibliographie}

Amariglio (S.) et Imbert (A.) (1980). - Enquête sur la teneur en nitrate-nitrite de quelques produits laitiers. Ann. Nutr. Alim., 34, 1053-1060.

Amariglio (S.) (1982). - Réglementation. Harmonisation des méthodes d'analyse des nitrates sur le plan international. Bull. Adria, 3, 6-7.

Barbieri (G.), Sala (G.), Gavioli (E.) e Beneventi (G.) (1979). - Sulla determinazione dei nitriti e nitrati nelle carni. Boll. dei Chim. dei Labor. Provinc., 5, 611-616.

Bonnett (R.) and HolleyHead (R.) (1974). - The interaction of nitrite with amino acid and peptide dérivatives: the ${ }^{15} \mathrm{~N}$-nuclear magnetic resonance approach. I.A.R.C., 9, 107-110.

Branca (P.), Ricottilli (F.) e Spagnolini (G.) (1980). - Sulla determinazione polarografica dei nitrati nei prodotti carnei e in altri alimenti. Boll. dei Chim. dei Labor. Provinc., 6, 385-395.

Bruget (P.) (1980). - Nitrates et nitrites dans les tissus végétaux. Méthodes analytiques. Ann. Nutr. Alim., 34, 757-764.

Cassens (R. G.), Woolford (G.), Lee (S. H.) and Goutefongea (R.) (1976). - Fate of nitrite in meat. Proc. 2nd Int. Symp. Nitrite Meat Prod. Zeist, p, 95.

CHoI (K.) and Fung (K.) (1980). - Determination of nitrate and nitrite in meat products by using a nitrate ion selective electrode. Analyst., 105, 241-245.

ChоріN (C.) (1982). - Interactions protéines-nitrates entre les différentes formes d'oxyde d'azote et la caséine bovine : mise en évidence, paramètres de fixation et aspects méthodologiques. Mémoire Ingénieur E.N.S.B.A.N.A.

CHOPin (C.) (1983). - Résultats non publiés.

Cox (J.A.) and BRAJTER (A.) (1979). - Selective electrocatalytic method for the determination of nitrite. Anal. Chem., 51, 2230-2232.

Cox (J. A.) and LiTwinsky (G.) (1979). - Voltametric ion selective electrode for the determination of nitrate. Anal. Chem., 51, 554-556.

Cox (R.D.) (1980). - Determination of nitrate and nitrite at the parts per billion level by chemiluminescence. Anal. Chem., 52, 332-335.

CSERhati (T.) (1973). - A method of nitrate determination using m-xylenol. In Dairy Sci. Abstr., 35, n ${ }^{\circ} 1499$.

De Saint-Blanquat (G.) (1980). - Aspects toxicologiques et nutritionnels des nitrates et des nitrites. Ann. Nutr. Alim., 34, 827-864.

Deschamps (P.) (1980). - Les teneurs en nitrites et en nitrates des aliments prélevés au cours de l'inventaire national de la qualité alimentaire. Ann. Nutr. Alim., 34, 901-908.

Fritsch (P.), Klein (D.) et De Saint-Blanquat (G.) (1980). - Excrétion salivaire et biliaire des nitrates chez le chien. Ann. Nutr. Alim., 34, 1089-1096. 
Frouin (A.), Thenot (M.), Jondeau (D.), Barraud (C.), Grimault (M. L.), Durand (P.) et Vendeuvre (J. L.) (1976 a). - Etudes sur l'état du nitrite dans les produits de viandes. Ann. Nutr. Alim., 30, 773-788.

Frouin (A.), Thenot (M.), Jondeau (D.), Patte (K.), Barraud (C.), Grimault (M. L.), Durand (P.) et Vendeuvre (J. L.) (1976 b). - Nitrates et nitrites. Révision nécessaire de nos conceptions et méthodes d'analyses. Ann. Fals. Exp. Chim., $69,629-635$.

Frouin (A.), JondeaU (D.), Bidard (J. P.) et Joannes (M.) (1980). - Dosage des nitrates et des nitrites dans les produits animaux. Ann. Nutr. Alim., 34, 765-778.

Funazo (K.), TANAKa (M.) and Shono (T.) (1980). - Determination of nitrite at parts per billion levels by derivatization and electron capture gas chromatography. Anal. Chem., 52, 1222-1224.

Görner (F.), Hluchan (E.) und Antalike (E.) (1978). - Der Nitrat und Nitritgehalt getrockneter Milchprodukte. Dtsch. Molk. Ztg., 99, 1584-1586.

Goutefongea (R.), Cassens (R. G.) and Woolford (G.) (1977). - Distribution of sodium nitrite in adipose tissue during curing. J. Food. Sci., 42, 1637-1640.

Guingamp (M. F.), Schwartz (A.) et Linden (G.) (1978). - Teneurs en nitratesnitrites des lactosérums de fromagerie : aspect méthodologique et causes de variation. Le Lait, 577, 371-380.

Guingamp (M. F.) et Linden (G.) (1980). - Influence des traitements technologiques sur les teneurs en nitrates-nitrites du lait. Ann. Nutr. Alim., 34, 1061-1067.

GuingamP (M. F.) et Linden (G.) (1981). - Résultats non publiés.

Guingamp (M. F.), Linden (G.) et Alais (C.) (1982). - Variation de la concentration en nitrates des produits laitiers au cours des procédés technologiques. XXI internat. Dairy Congr., vol. 1, b.2, 182.

Guingamp (M. F.) et Linden (G.) (1983). - Réactivité des nitrites. Incidences physiopathologiques. D. Réactivité du nitrite sur la liaison peptidique. Compte rendu D.D.S.T.I., 51-61.

Hamilton (J. E.) (1976), - Collaborative study of the colorimetric determination of nitrate and nitrite in cheese. J.A.O.A.C., 59, 284-288.

Harding (F.) and GREgSON (R.) (1978). - Nitrates and nitrites in skim milk and whey powders in the United Kingdom. XX Internat. Dairy Congr., E. 356-357.

Huynh (C.), Jacquet (J.) et Silberzahn (P.) (1969). - Dosage des nitrates et des nitrites par une méthode polarographique en courant alternatif superposé. Ind. Alim. Agr., 86, 1439-1443.

IskANDARANi (Z.) and PLETRZYK (D.) (1982). - Determination of nitrite and nitrate in water and food samples by ion interaction chromatography. Anal. Chem., $54,2601-2603$.

Ito (T.), Cassens (R. G.) and Greaser (M. L.) (1979). - Reaction of nitrite with trytophyl residues of protein. J. of Food Sci., 44, 1144-1149.

KANEDA (Y.), KANAMORI (T.) and IWAïDA (M.) (1977). - Nitrate determination in cheese, by use of ion selective electrode. J. Hyg. Chem., 23, 301-306.

Mc KAY (A. J.) (1974). - The determination of nitrate and nitrite in cheese. Aust. J. Dairy Technol., 29, 34-36.

Keshavarz-Dehno (A.) (1979), - Influence des micro-organismes sur la synthèse des nitrosamines dans les produits fromagers. Thèse Doctorat Spé., Univ. Nancy.

Kiang (C.), Kuan (S.) and Guilbault (G.) (1978 a). - Enzymatic determination of nitrate: electrochemical detection after reduction with nitrate reductase and nitrite reductase. Anal. Chem., 50, 1319-1322. 
Kiang (C.), Kuan (S.) and Guilbault (G.) (1978 b), - Enzymatic determination of nitrate: fluorimetric detection after reduction with nitrate reductase. Anal. Chem., 50, 1323-1325.

Klein (D.), Gaconnet (N.), Beaufrand (M. J.) et Debry (G.) (1976). - Formation des nitrosamines dans le tractus digestif humain. Evolution du $\mathrm{pH}$ et des concentrations en nitrates et nitrites dans le suc gastrique au cours de la digestion. An. Nutr. Alim., 30, 813-821.

Klein (D.), Gaconnet (N.), Poullain (B.) et Debry (G.) (1978) . - Effet d'une charge en nitrate sur le nitrite salivaire et gastrique chez l'homme. $F d$. Cosmet. Toxicol., 16, 111-115.

KLeIN (D.) et Debry (G.) (1980). - L'analyse des nitrosamines volatiles dans les aliments et les boissons. Validité des résultats obtenus. Ann. Nutr. Alim., 34, 779-790.

Klein (D.), Keshavarz (A.), Lafont (P.), Hardy (J.) et Debry (G.) (1980). - Formation de nitrosamines dans les produits fromagers. Ann. Nutr. Alim., 34, $1077-1088$

KLEIN (D.) (1982). - Dosage des nitrates et des nitrites dans les laits et produits laitiers. Bull. Adria, 3, 3-4.

Knowles (M. E.), Mc Weeny (D. J.), Couchman (L.) and Thorogood (M.) (1974). Interaction of nitrite with proteins at gastric pH. Nature, 247, 288-289.

KREISNER (M.) und FINK (J.) (1978). - Ein Vergleich der Nitratbestimmungsmethoden in Milchprodukten. Deutsche Landwirtschafts Geselschaft, $36 \mathrm{p}$.

Kubberod (G.), Cassens (R. G.) and Greaser (M. L.) (1974). - Reaction of nitrite with sulfydryl groups of myosin. J. Food. Sci., 39, 1228-1230.

KURECHI (T.) and KikUGAWA (K.) (1979). - Nitrite-lipid reaction in aqueous system: inhibitory effects on nitrosamine formation. J. Food Sci., 44, 12631271.

Lange (E.) (1981). - Nitratbestimmung in Produkten aus Möhren mit einer nitratsensitiven Elektrode. Lebensm. Indus., 28, 269-272.

LASKowski (K.) and Bierska (J.) (1974 a). - Dosage de la teneur en nitrites dans le lait. XIX Internat. Dairy Congr., 1E, 473-474.

LASKOWSKI (K.) and BIERSKA (J.) (1974 b). - Determination of nitrates, nitrites and nitrosamines in cheeses. XIX Internat. Dairy Congr., 1E, 514-515.

LiedTKe (M.) and MeLoAN (C.) (1976). - Rapid screening determination of nitrate in baby food using the nitrat selective electrode. J. Agric. Food. Chem., 24, 410-412.

Lhuissier (M.), Suschetet (M.) et Causeret (J.) (1976). - Influence des nitrites et des nitrates sur certains aspects de l'état de nutrition vitaminique. Ann. Nutr. Alim., 30, 847-858.

Lowy (R.) et Manchon (P.) (1976). - Effets toxiques divers des nitrates et des nitrites. Ann. Nutr. Alim., 30, 839-845.

Mahieu (H.), Luouet (F. M.) et Mouillet (L.) (1976-1977). - A propos de la teneur des laits individuels et de mélange en matière minérale et urée, Le Lait, (56), 657-698; (57), 55-112 et 184-214.

Mahieu (H.), Luquet (F. M.), Mouillet (L.) et Boudier (J. F.) (1980). - Présence de nitrates et de nitrites dans le lait. Evaluation de la teneur des laits français en nitrates-nitrites : enquêtes 1973, 1974, 1975, 1979. Ann. Nutr. Alim., 34, 1045-1052.

Mahieu (H.), Luquet (F. M.), Mouillet (L.) et Boudier (J. F.) (1982). - Nitratesnitrites. Evaluation de la teneur des laits français. Enquêtes 1973, 1974, 1975, 1979. XXI Internat. Dairy Congr., vol. 1, B. 2, 581. 
MAnning (P. B.), Coulter (S. T.) and Jenness (R.) (1968). - Determination of nitrate and nitrite in milk and dry products. I. Dairy Sci., 51, 1725-1730.

Melet (J. J.) Morlot (M.), Passavy (J.) et Salado (J.) (1980). - Hydrogéologie et médecine dans l'approche des problèmes de santé liés aux nitrates : exemples en Lorraine. Ann. Nutr. Alim., 34, 969-978.

Molt (N.) (1983) . - Nouveaux procédés d'isolement et de synthèse des composés d'Amadori. Applications. Thèse doct. Etat, Univ. Nancy I.

Mrowetz (G.) und Klostermeyer (H.) (1977 a). - Bestimmung des Nitratgehaltes getrockneter Milchprodukte. II. Schnellbestimmung mit Diphenylamin und Vergleich mit der Cadmium-Reduktionsmethode. Milchwissenschaft, 32, 705-708.

Mrowetz (G.) und Klostermeyer (H.) (1977 b). - Bestimmung der Nitratgehaltes getrockneter Milchprodukte. I. Bestimmung nach Reduktion mit metallischem Cadmium. Milchwissenschaft, 32, 647-650.

Nakat (H.), Cassens (R. G.), Greaser (M. L.) and Woolford (G.) (1978). - Significance of the reaction of nitrite with tryptophan. J. Food Sci., 43, 18571860.

NiJHUis (H.), HeEschen (W.) and Blüthgen (A.) (1979). - Automated determination of nitrate and nitrite in milk and dairy products by means of the continuous flow analysis. Milchwissenschaft, 34, 414-416.

Nijhuis (H.), HeEschen (W.), Blüthgen (A.) and Tolle (A.) (1982). - Sur la présence de nitrate et nitrite dans le lait et les produits laitiers. XXI Internat. Dairy Congr., vol. 1, B. 2, 565.

NORme Internat. provisoire F.I.L. 95 (1980). - Lait sec. Détermination des teneurs en nitrates et en nitrites. Méthode par réduction au cadmium et photométrie.

Norme Internat. PRovisoire F.I.L. 96 (1980). - " Fromage de sérum » (Whey cheese). Détermination des teneurs en nitrates et en nitrites. Méthode par réduction au cadmium et photométrie.

Norme Internat. PRovisoire F.I.L. 97 (1980). - Poudre de sérum. Détermination des teneurs en nitrates et en nitrites. Méthode par réduction au cadmium et photométrie.

PFeiffer (S. L.) and SMith (J.) (1975). - Nitrate determination in baby food, using the nitrate ion selective electrode. J.A.O.A.C., 58, 915-919.

PoLLOCK (J. R. A.) (1981). - Nitrosation products from peptides. VII Internat. meeting on analysis and formation of nitroso compounds, Tokyo.

PRZYBylowski (P.) and KISZA (J.) (1982). - Changes in the contents of nitrates and nitrites during the production and storage of milk powder. XXI Internat. Dairy Congr., vol, 1, B. 2, 38.

Remond (B.) (1975). - La teneur du lait de vache en nitrate. Le Lait, 55, 390-395.

RESMini (P.) and Volonterio (G.) (1973). - Nitrate content of milk. Latte, 9, 167-169.

RoTHERY (G. B.) (1968). - Reduction of nitrite content of products dried in direct-fired spray dryers. Aust. I. Dairy Technol., 23, 76-81.

Rougie (P.), Noel (P.) et Goutefongea (R.) (1980). - Influence des conditions de stockage et de traitement des échantillons sur le " nitrite libre " dans les produits carnés. Ann. Nutr. Alim., 34, 1009-1018.

SEN (N. P.), CARR (P.) and KLATT (L.) (1976). - Determination of nitrate ion at the part per billion level in environmental samples with a continuous flow immobilized enzyme reaction. Anal. Chem., 48, 954-958.

Sen (N.P.), Donaldson (B.A.), Seaman (S.), Iyengar (J.R.) and Miles (W. F.) (1978). - Recent studies in Canada on the analysis and occurrence of volatil $\mathrm{N}$-nitrosocompounds. Environmental aspect of $\mathrm{N}$-nitrosocompounds. I.A.R.C., 19, 373-390. 
SöDERHJELm (P.) (1982). - Determination of nitrate in some dairy products by high pressure liquid chromatography. XXI Internat. Dairy Congr., vol. 1, B. 2, 249.

SweEtsur (A.) and Wilson (A.) (1975). - An ion selective electrode method for the determination of nitrate in grass and clover. Analyst., 100, 485-488.

Tanaka (A.), Nose (N.), Yamada (F.), Saito (S.) and Watanabe (A.) (1981), - Determination of nitrite in human, cow and market milks by gas liquid chromatography with electron capture detection. J. Chromatogr., 206, 531-540.

TANAKA (A.), NOSE (N.) and IWASAKI (H.) (1982). - Determination of nitrate in meat products and cheeses by gas liquid chromatography with electron capture detection. J. Chromatogr., 235, 173-185.

Toyoda (M.), Suzuki (H.), Ito (Y.) and INAïDa (M.) (1978). - Gas liquid chromatography determination of nitrate and nitrite in cheese, ham, fish, sausage, cod roes and salmon roes. J.A.O.A.C., 61, 508-512.

Usher (C.) and Telling (G.) (1975). - Analysis of nitrate and nitrite in food stuffs: a critical review. J. Sci. Fd. Agric., 26, 1793-1805.

Voilliot (J. P.), Mere (C.) et Degrimal (J.P.) (1980). - Evolution des teneurs en nitrates dans les eaux d'alimentation du département de l'Yonne : nouveau bilan depuis 1975. Ann. Nutr. Alim., 34, 989-996.

Walters (C. L.), Hart (R. J.), Keefer (L. K.) and Newberne (P. M.) (1980). The sequential determination of nitrite, N-nitrosocompounds and nitrate and its application. I.A.R.C., 31, 389-402.

WHEELER (W. H.) (1980) . - Chemical and engineering aspects of low $\mathrm{NO}_{\mathrm{x}}$ concentration. Chem. Engineer., 362, 693-699.

Woolford (G.), Cassens (R. G.), Greaser (M. L.) and Sebranek (J. G.) (1976). The fate of nitrite: reaction with protein. J. Food. Sci., 41, 585-588.

Woolford (G.) and Cassens (R. G.) (1977). - The fate of sodium nitrite in bacon. J. Food. Sci., 42, 586-596.

WU (W. S.) and SASCHENBRECKER (P. W.) (1977). - Nitration of benzene as method for determining nitrites and nitrates in meat products. J.A.O.A.C., 60, 11371141.

ZAUGG (H. E.) (1961). - Nitrosation and diazonium salt coupling of Amadori products. J. Organic. Chem., 26, 2718-2720.

ZimmermanN (K.) und Von Lengerken (J.) (1980a). - Zur Anwendung einer neuen $\mathrm{NO}_{3}$ sensitiven Elektrode in Futtermitteln. Die Nahrung, 24, 197-199.

Zimmermann (K.) und Von Lengerken (J.) (1980 b). - Nitratbestimmung in Silagen mit ionensensitiven Elektroden. Monatsh. für veterinarmedizin, 35, 100-101. 